\title{
Nonlinear Bremsstrahlung*
}

\author{
R. K. Osborn \\ Department of Nuclear Engineering, University of Michigan, Ann Arbor, Michigan 48105 \\ (Received 27 September 1971)

\begin{abstract}
A computation of the probability per unit path per photon for an arbitrary number of photons to be emitted or absorbed by an electron passing through the field of an ion is presented. The results provide a qualitative estimate of the conditions for the onset of nonlinear processes.
\end{abstract}

The absorption of laser radiation by nonlinear, inverse bremsstrahlung may play a significant role in the heating of laser produced plasmas. Cross sections describing the phenomenon have been variously derived. ${ }^{1-3}$ However, photon absorption coefficients do not appear to have been obtained from these results. (A derivation of a photon absorption coefficient based upon some unpublished work by Schoefield is described by Kidder. ${ }^{4}$ ) In this paper we describe an alternate derivation leading to quantitatively different, though apparently qualitatively similar, results. Although repititious, the derivation of the cross section will be briefly sketched for completeness because the scheme used here is different than those described in the aforementioned references.

In Sec. I the system is modeled and a formula for a cross section to describe the simultaneous emission or absorption of an arbitrary number of photons by an electron passing through the field of an ion is obtained. In Sec. II, a formula for net photon absorption coefficients is presented. In Sec. III the results of Sec. II are briefly summarized and discussed.

\section{MODEL AND FORMULA}

The Hamiltonian for the system is taken to be

$$
\begin{aligned}
& H=p^{2} / 2 m-Z e^{2} / r+H^{R}+H_{1}^{P R}+H_{2}^{P R}, \\
& H^{R}=\hbar \omega \alpha^{\dagger} \alpha, \\
& H_{1}^{P R}=-(e / m c) \overrightarrow{\mathrm{A}} \cdot \overrightarrow{\mathrm{P}}, \\
& H_{2}^{P R}=\left(e^{2} / 2 m c^{2}\right) A^{2}, \\
& A=c\left(2 \pi \hbar / L^{3} \omega\right)^{1 / 2} \vec{\epsilon}\left(\alpha^{\dagger}+\alpha\right) .
\end{aligned}
$$

The ion is presumed infinitely massive and located at the origin. The Coulomb potential should be regarded as Debye-shielded; but because the Debye length is so large compared to the wavelength of the electrons, such shielding plays no significant role in the subsequent analysis. It has already played a role, however, in our modeling of the system. For the densities $\left(n \gtrsim 10^{18} / \mathrm{cm}^{3}\right)$ and temperatures $(k T \lesssim 200 \mathrm{eV})$ of present concern, the Debye length is of the order of, or less than, $10^{-5}$ $\mathrm{cm}$. This provides some justification for model- ing the electromagnetic field in the dipole approximation [Eq. (1e)]. The operators, $\alpha^{\dagger}$ and $\alpha$, are the conventional $1^{5}$ creation and annihilation operators of quantum electrodynamics, and $L^{3}$ is the volume of the quantization box. The laser field is assumed to be pure, hence only one frequency $(\omega)$ and polarization $(\epsilon)$ appears in the expression for the vector potential $\vec{A}$.

In order to obtain relatively simple analytic results, we treat the Coulomb potential as a perturbation rather than using the proper continuum Coulomb eigenfunctions. Thus the quantity to be computed is the transition probability per unit time, i.e.,

$$
T_{K^{\prime} \eta^{\prime}, K \eta}=(1 / \tau)\left|\left\langle K^{\prime} \eta^{\prime}\left|e^{-i H \tau / \hbar}\right| K \eta\right\rangle\right|^{2},
$$

in the limit of small $\tau$, where the states $|K \eta\rangle$ are eigenstates of the electron kinetic energy and of the free-radiation field, e.g. ,

$$
\begin{aligned}
& \frac{p^{2}}{2 m}|K \eta\rangle=\left(\hbar^{2} K^{2} / 2 m\right)|K \eta\rangle=E_{K}|K \eta\rangle, \\
& H^{R}|K \eta\rangle=\hbar \omega \eta|K \eta\rangle .
\end{aligned}
$$

The present analysis is therefore approximate in the same sense as Ref. 3. In that reference, however, it was found (the radiation field was treated classically) that $H_{2}^{P R}$ contributed nothing to the computation of the cross section. Therefore, this term will be arbitrarily ${ }^{6}$ ignored here as a probably unnecessary complication. Then, to first order in the Coulomb potential, the transition probability of Eq. (2) becomes

$$
\begin{aligned}
& T_{K^{\prime} \eta^{\prime}} K \eta \simeq \frac{1}{\tau}\left|\frac{4 \pi Z e^{2}}{\left|\overrightarrow{\mathrm{K}}-\overrightarrow{\mathrm{K}}^{\prime}\right|^{2}}\right|^{2} \\
& \quad \times\left|\int_{0}^{\tau} d t e^{i t\left(E_{K^{\prime}}-E_{K^{\prime}}\right) / \hbar}\left\langle\eta^{\prime}\left|U^{R^{\prime} \dagger} U^{R}\right| \eta\right\rangle\right|^{2},
\end{aligned}
$$

where explicit use has been made of the fact that our eigenstates are also eigenfunctions of momentum, and we have defined

$$
U^{R}=e^{-i t\left(H^{R}+H_{1}^{P R}\right) / \hbar},
$$

where now

$$
H_{1}^{P R}=-(e \hbar / m c) \overrightarrow{\mathrm{A}} \cdot \overrightarrow{\mathrm{K}} \text {. }
$$

The evaluation of the matrix element in Eq. (4) 
is tedious but straightforward, leading to (setting $\left.\eta^{\prime}=\eta \pm n\right)$

$$
\begin{aligned}
\langle\eta \pm n| U^{R^{\prime}+} & U^{R}|\eta\rangle \\
& =e^{h} \sum_{r=0}^{\infty} \frac{a^{*}\left(\overrightarrow{\mathrm{K}}, \overrightarrow{\mathrm{K}}^{\prime}\right)^{r} a\left(\overrightarrow{\mathrm{K}}^{\prime}, \overrightarrow{\mathrm{K}}\right)^{r \mp n}(n+r) !}{r !(r \mp n) ![\eta !(\eta \pm n) !]^{1 / 2}},
\end{aligned}
$$

where

$$
\begin{aligned}
& h=g-q q^{\prime *} \text {, } \\
& g=\left(\beta^{2} / \hbar^{2} \omega^{2}\right)\left[1-i \omega t-e^{-i \omega t}\right] \\
& +\left(\beta^{2} / \hbar^{2} \omega^{2}\right)\left[1+i \omega t-e^{i \omega t}\right], \\
& q=(\beta / \hbar \omega)\left(e^{-i \omega t}-1\right), \\
& \beta=-\left(\frac{2 \pi e^{2} \hbar^{3}}{m^{2} L^{3} w}\right)^{1 / 2} \vec{E} \cdot \overrightarrow{\mathrm{K}},
\end{aligned}
$$

and

$$
a\left(\overrightarrow{\mathrm{K}}^{\prime}, \overrightarrow{\mathrm{K}}\right)=\left(\beta^{\prime}-\beta\right)\left(1-e^{-i \omega t}\right) / \hbar \omega
$$

Because of the dependence of $\beta$ upon $L^{-3 / 2}, h$ is small and can be neglected. The quantities $a$ and $a^{*}$ are then expanded in power series in $e^{i \omega t}$ and only the lowest power retained. One then finds that

$$
\begin{aligned}
& \left\langle\eta \pm n\left|U^{R^{\prime} t} U\right| \eta\right\rangle \\
& \simeq \frac{e^{ \pm i n \omega t}}{[\eta !(\eta \pm n) !]^{1 / 2}} \sum_{r=0}^{\infty} \frac{(-1)^{r} x^{2 r \mp n}(\eta+r) !}{r !(r \mp n) !},
\end{aligned}
$$

where

$$
x=\left(\beta^{\prime}-\beta\right) / \hbar \omega .
$$

Because $\eta$ is so large (it represents the number of photons in the laser field), the series in Eq. (13) can be well approximated by a Bessel function. Consequently, the matrix element can be simplified to read

$$
\left\langle\eta \pm n\left|U^{R^{\prime} \dagger} U^{R}\right| \eta\right\rangle \simeq e^{ \pm i n \omega t} J_{\mp n}(\sqrt{n} x) .
$$

Our transition probability per unit time now reads

$$
\begin{aligned}
T_{K^{\prime} \eta \pm n, K \eta} \simeq \frac{2 \pi}{\hbar} J_{n}^{2}(\sqrt{\eta} x)\left|\frac{4 \pi Z e^{2}}{\left|\overrightarrow{\mathrm{K}}-\overrightarrow{\mathrm{K}}^{\prime}\right|^{2}}\right|^{2} \\
\times \delta\left(E_{K^{\prime}}-E_{K} \pm n \hbar \omega\right),
\end{aligned}
$$

which leads us to the cross section

$$
\begin{aligned}
& \sigma_{\eta \pm n}\left(\overrightarrow{\mathrm{K}}, \overrightarrow{\mathrm{K}}^{\prime}\right) d^{3} K^{\prime} \\
& =\left(\frac{2 Z e^{2}}{\hbar}\right)^{2} \frac{m}{K} \frac{J_{n}^{2}(\sqrt{\eta} x) d^{3} K^{\prime}}{\left|\overrightarrow{\mathrm{K}}-\overrightarrow{\mathrm{K}}^{\prime}\right|^{4}} \\
& \quad \times \delta\left(E_{K^{\prime}}-E_{K} \pm n \hbar \omega\right) .
\end{aligned}
$$

This formula is identical to the one obtained by Brehme. ${ }^{3}$

\section{PHOTON ABSORPTION COEFFICIENTS}

The reaction rate $/ \mathrm{cm}^{3}, R_{\eta \pm n}$, for the simultaneous emission or absorption of $n$ photons is computed according to

$$
R_{\eta \pm n}=n^{e} n^{I} \iint d^{3} K d^{3} K^{\prime}(\hbar K / m) P(\overrightarrow{\mathrm{K}}) \sigma_{\eta \pm n}\left(\overrightarrow{\mathrm{K}}, \overrightarrow{\mathrm{K}}^{\prime}\right),
$$

where $P(\overrightarrow{\mathrm{K}})$ is an electron momentum distribution (here taken to be Maxwellian) and $n^{e}$ and $n^{I}$ are the electron and ion densities, respectively. Most of the integrations can be carried out to yield

$$
R_{\eta \pm n}=4\left(\frac{m}{2 \pi \Theta}\right)^{1 / 2} \frac{n^{e} n^{I} Z^{2} e^{4} \gamma^{2}}{\hbar^{2}} e^{\mp n \hbar \omega / 2 \Theta} H_{n},
$$

where

$$
H_{n} \equiv 2 \pi \int_{0}^{\infty} d q \int_{-1}^{1} d \mu \frac{e^{-\left(q^{2}+6_{n}^{2} / q^{2}\right) / \zeta^{2}} J_{n}^{2}(q \mu)}{q^{3}},
$$

and we have introduced the notations

$$
\begin{aligned}
& \gamma^{2}=2 \pi e^{2} \hbar f / m^{2} \omega^{3}, \\
& f=\eta / L^{3}, \quad \zeta^{2}=8 m \Theta \gamma^{2} / \hbar^{2}, \\
& \delta_{n}=2 m \gamma^{2} \omega n / \hbar, \quad \Theta=k_{B} T .
\end{aligned}
$$

The net energy-absorption coefficient is now obtained according to

$$
k=\sum_{n=1}^{n} \frac{n}{c f}\left(R_{\eta-n}-R_{\eta+n}\right) \equiv k_{w} F(\zeta, y),
$$

where $k_{w}$ is the weak-field absorption coefficient given by

$k_{w}=\frac{8 \pi}{3}\left(\frac{2 \pi m}{\Theta}\right)^{1 / 2} \frac{e^{2}}{\hbar c} \frac{n^{e} n^{I} r_{e}^{2} Z^{2} C^{4}}{\omega^{3}} \sinh y K_{0}(y)$,

where

$$
y=\hbar \omega / 2 \theta, \quad r_{e}^{2}=e^{2} / m c^{2},
$$

and $K_{0}$ is a modified Bessel function of the second kind.

\section{SUMMARY AND DISCUSSION}

The function $F(\zeta, y)$ appearing in Eq. (21) is a measure of the multiphoton, or nonlinear, effects on the net absorption coefficient. The quantity, $k_{w}$, is the usual [ e.g., employed by Dawson ${ }^{7}$ provided his $\ln \Lambda$ is identified as $\left.(1 / 2 y)(\sinh y) K_{0}(y)\right]$ linear, or single-photon absorption coefficient. The parameter $\zeta^{2}$ may be written as

$$
\zeta^{2}=\frac{8 \pi}{y} \frac{e^{2}}{\hbar c} \frac{I}{m \omega^{3}}
$$

where $I$ is the photon energy flux in ergs $/ \mathrm{cm}^{2} / \mathrm{sec}$. It is convenient therefore to display $F$ in alternate forms; one convenient for examining the onset of nonlinear effects at low intensities, and the other appropriate to asymptotic approximation for high 
intensity. The representation of $F$ as a power series in $\zeta^{2}$ is

$$
\begin{aligned}
F= & \frac{3}{(\sinh y) K_{0}(y)} \sum_{n} n \sinh n y \\
& \times \sum_{k=0}^{\infty} \frac{(-1)^{k}\left(n y \zeta^{2}\right)^{n+k-1}(2 n+2 k) ! K_{n+k-1}(n y)}{8^{n+k-1}(2 n+2 k+1)(2 n+k) ![(n+k) !]^{2}} \\
= & 1-\frac{3 y \zeta^{2} K_{1}(y)}{40 K_{0}(y)}+\frac{3 y \zeta^{2} \sinh 2 y K_{1}(2 y)}{40 \sinh y K_{0}(y)}+O\left(\zeta^{4}\right) .
\end{aligned}
$$

For small $\hbar \omega / 2 k_{B} T$, the series to order $\zeta^{4}$ reduces to

$$
F \simeq 1-\frac{9 y^{2} \zeta^{2}}{80}=1-\frac{9 \pi}{10} y \frac{e^{2}}{\hbar c} \frac{I}{m \omega^{3}} .
$$

This result is in good qualitative agreement with the one discussed by Kidder. ${ }^{4}$

The form of $F$ that is suitable for asymptotic examination is

$$
F=\frac{3 / \pi}{\sinh y K_{0}(y)} \sum_{n} n \sinh n y H_{n},
$$

where $H_{n}$ is given in Eq. (20). For large $\zeta$, the functions $H_{n}$ can be approximately displayed as

$$
H_{n} \approx \frac{8 \pi^{3 / 2} C_{n} e^{-n y}}{\zeta^{3}(n y)^{2}}\left(1+\frac{1}{n y}\right)+O\left(\zeta^{-4}\right),
$$

where

$$
C_{n} \equiv \int_{0}^{\infty} d \alpha J_{n}^{2}(\alpha)
$$

Noting that $H_{n}$ is a rapidly decreasing function of increasing $n$, we surmise that most of the contribution to Eq. (25) will come from the first couple of terms in the sum. In that case, we find that

$$
F \sim \frac{3 / \pi}{\sinh y K_{0}(y)}\left[H_{1} \sinh y+H_{2} \sinh 2 y+\cdots\right] .
$$

For small $y$, Eq. (28) reduces to

$F \sim \frac{3}{\pi}\left(\frac{m c \theta \omega^{2}}{e^{2} I}\right)^{3 / 2} \frac{1}{K_{0}(y)}\left(C_{1}+\frac{C_{2}}{4}+\cdots+O\left(\zeta^{-1}\right)\right)$.

This result is also in good qualitative agreement with the one discussed by Kidder. ${ }^{4}$ However, it appears to disagree considerably with the result derived by Hughes and Nicholson-Florence ${ }^{8}$; though it is similar to the result they quote as obtainable from Rand. ${ }^{1}$ In the present instance, the asymptotic absorption coefficient is [recall Eq. (21)]

$$
k \sim 4(2 \pi)^{1 / 2} \frac{m^{2} n^{e} n^{I} r_{e}^{2} Z^{2} c^{9 / 2} \omega}{e I^{3 / 2}}\left(C_{1}+\frac{C_{2}}{4}+\cdots\right),
$$

i.e., it is proportional to the frequency of the incident light, insensitive to temperature, and inversely proportional to the light intensity to the three-halves power.
*Work partially supported by the Air Force Office of Scientific Research under Contract No. AFOSR-69-1693 and by the Paul Goebel Chair of Advanced Technology, College of Engineering, University of Michigan.

${ }^{1}$ S. Rand, Phys. Rev. 136, B231 (1964).

${ }^{2}$ F. V. Bunkin and M. V. Federov, Zh. Eksperim. i Teor. Fiz. $\underline{49}, 1215$ (1965) [Sov. Phys. JETP 22, 844 (1966)].

${ }^{3}$ Helmut Brehme, Phys. Rev. C 3, 837 (1971).

${ }^{4}$ Ray E. Kidder, University of California Report No. UCRL-71775 (unpublished).

${ }^{5} \mathrm{~W}$. Heitler, The Quantum Theory of Radiation (Oxford U.P., London, 1954).

${ }^{6}$ The use of the term "arbitrary" is perhaps a bit too strong; since, as is seen in Eq. (17), the cross sections obtained here by the neglect of $H_{2}^{P R}$, and some other ap- proximations, are identical to those of Ref. 3 in which the radiation field is treated classically $a b$ initio. That a classical treatment of these intense electromagnetic fields seems acceptable is also buttressed by the approaches to related problems described in Refs. 1,2 , 4 , and 8. In fact, the whole of Sec. I could have been omitted-it was inserted only because the cross-section derivation sketched here is different from the one of Ref. 3. The neglect of peculiarily quantum corrections here is probably much less serious than the use of plane waves to describe the electrons in the initial and final states. See also Norah V. Cohan and Hendrick F. Hameka, Phys. Rev. 151, 1076 (1966).

7John M. Dawson, Phys. Fluids ㄱ, 981 (1964).

${ }^{8}$ T. P. Hughes and M. B. Nicholson-Florence, J. Phys. A 1,588 (1968). 